Georgian Mathematical Journal

Volume 11 (2004), Number 3, 449-466

\title{
BADORA'S EQUATION ON NON-ABELIAN LOCALLY COMPACT GROUPS
}

\author{
E. ELQORACHI, M. AKKOUCHI, A. BAKALI, AND B. BOUIKHALENE
}

\begin{abstract}
This paper is mainly concerned with the following functional equation

$$
\int_{G}\left\{\int_{K} f(x t k \cdot y) d k\right\} d \mu(t)=f(x) f(y), \quad x, y \in G,
$$

where $G$ is a locally compact group, $K$ a compact subgroup of its morphisms, and $\mu$ is a generalized Gelfand measure. It is shown that continuous and bounded solutions of this equation can be expressed in terms of $\mu$-spherical functions. This extends the previous results obtained by Badora (1992) on locally compact abelian groups. In the case where $G$ is a connected Lie group, we characterize solutions of the equation in question as joint eigenfunctions of certain operators associated to the left invariant differential operators.
\end{abstract}

2000 Mathematics Subject Classification: 39B05, 43A90.

Key words and phrases: D'Alembert functional equation, Gelfand measure, $\mu$-spherical function, Lie group.

\section{INTRODUCTION}

Let $G$ be a locally compact group, $K$ a compact subgroup of morphisms of $G$. The action of $k \in K$ on $x \in G$ is denoted by $k \cdot x$, and the normalized Haar measure on $K$ by $d k$. Furthermore for a complex bounded measure $\mu$ on $G$, i.e., $\mu \in M(G)$, the topological dual of $C_{0}(G)$, the Banach space of continuous functions vanishing at infinity (cf. [6], 13.1.2 and 13.20.1), $\check{\mu}$ (resp. $\mu_{x}$ ) denotes the measure defined by $\langle\check{\mu}, f\rangle=\int_{G} f\left(t^{-1}\right) d \mu(t)$ (resp. $\left\langle\mu_{x}, f\right\rangle=$ $\left.\int_{G} f\left(t x^{-1}\right) d \mu(t)\right)$ for all continuous and bounded functions $f$ on $G$.

In the paper [4] Badora considered the following functional equation

$$
\int_{G}\left\{\int_{K} f(x t k \cdot y) d k\right\} d \check{\mu}(t)=f(x) f(y), \quad x, y \in G,
$$

when $G$ is abelian and $\mu$ is $K$-invariant, i.e., $\int_{G} f(k \cdot t) d \mu(t)=\int_{K} f(t) d \mu(t)$ for all continuous functions with compact support on $G(f \in \mathcal{K}(G))$ and for all $k \in K$. The essentially bounded non-zero solutions of equation (1.1) are completely defined as

$$
f(x)=\int_{K}\left(\chi * \mu_{k \cdot x}\right)(e) d k, \quad x \in G,
$$


where $\chi$ is a character of $G$ and $e$ is the identity element of the abelian group $G$ (see [4]).

In the paper we are going to study the functional equation

$$
\int_{G}\left\{\int_{K} f(x t k \cdot y) d k\right\} d \mu(t)=f(x) f(y), \quad x, y \in G .
$$

This category contains not only equations of the from

$$
\int_{K} f(x k \cdot y) d k=f(x) f(y), \quad x, y \in G
$$

(see [4], [5], [15], [16] and [17]), but also the generalized d'Alembert's functional equation

$$
\int_{G} f(x t y) d \mu(t)+\int_{G} f\left(x t y^{-1}\right) d \mu(t)=2 f(x) f(y) \quad x, y \in G,
$$

which was studied in [9].

Concerning continuous solutions of (1.4), one of the main results is due to Shin'ya [14]: any non-zero continuous solution of equation (1.4) has the form

$$
f(x)=\int_{K} \chi(k \cdot x) d k \quad \text { for all } x \in G,
$$

where $\chi: G \longrightarrow \mathbb{C} \backslash\{0\}$ is a continuous homomorphism of the abelian group $G$ (cf. [14], Corollary 3.12).

In Section 2 (Theorem 2.1), we give necessary and sufficient conditions for a (measurable) essentially bounded function $f$ to satisfy equation (1.3). One of these conditions is

$$
M_{K}(\check{\mu} * h * f)=\langle h, \check{f}\rangle f
$$

for all $h \in L_{1}(G, d x)$, where $M_{K}(h)(x)=\int_{K} h(k \cdot x) d k$ for all $h \in L_{\text {loc }}^{1}(G, d x)$ and for all $x \in G$. This explains why we restrict ourselves to solutions $f \in C_{b}(G)$.

In Theorem 2.2, we prove that the map

$$
f \longmapsto\langle f, \omega\rangle=\int_{G} f(x) \omega(x) d x
$$

is a continuous character of the Banach algebra $\mu * M_{K}\left(L_{1}(G, d x)\right) * \mu=M_{K}(\mu *$ $\left.L_{1}(G, d x) * \mu\right)$ if and only if $\omega$ is a non zero solution of the functional equation (1.3).

In Section 3, $\mu$ is a generalized Gelfand measure which is $K$-invariant. We extend the above-mentioned results due to Badora [4]. More precisely, under an additionally condition that every closed ideal of the commutative Banach algebra $\mu * L_{1}(G, d x) * \mu$ is contained in some maximal regular ideal, we find explicit formulas for solutions expressing them in terms of $\mu$-spherical functions (Theorem 3.1).

In the final part of our paper when $G$ is a connected Lie group and $\mu$ is an idempotent measure which is $K$-invariant, we characterize solutions of (1.3) as 
joint eigenfunctions of certain operators associated to the left invariant differential operators (Theorem 4.1).

Our notation is described in the following set-up and we will stick to it in the rest of the paper.

General set-up and notation. Let $G$ be a locally compact separable Hausdorff group, $e$ its identity element, $C(G)\left(\right.$ resp. $\left.C_{b}(G)\right)$ the complex algebra of all continuous (resp. continuous and bounded) complex-valued functions on $G$.

Let $K$ be a subgroup of the group $\operatorname{Mor}(G)$ of all mappings $k$ of $G$ onto itself that are either automorphisms and homeomorphisms $\left(k \in K^{+}\right)$or antiautomorphisms and homeomorphisms $\left(k \in K^{-}\right)$.

We assume that $K$ has a topology making it a compact Hausdorff group with the property that the canonical map $K \times G \longrightarrow G$ sending each pair $(k, x)$ onto $k \cdot x$ is continuous.

For any $k \in K$, and for any function $f$ on $G$, we put $(k \cdot f)(x)=f\left(k^{-1} \cdot x\right)$ and say that $f$ is $K$-invariant if $k \cdot f=f$ for all $k \in K$.

The algebra of all regular and complex bounded measures on $G$ is denoted by $M(G)$. We recall that the convolution of $M(G)$ is given by

$$
\langle\mu * \nu, f\rangle=\int_{G} \int_{G} f(t s) d \mu(t) d \nu(s), \quad f \in C_{b}(G) .
$$

For any $\mu \in M(G)$ and any $k \in K$, we put $\langle k \cdot \mu, f\rangle=\langle\mu, k \cdot f\rangle, f \in C_{b}(G)$, and say that $\mu$ is $K$-invariant if $k \cdot \mu=\mu$ for all $k \in K$.

A function $f \in C_{b}(G)$ is $\mu$-biinvariant if $f_{\mu}=f$, where $f_{\mu}$ is the continuous and bounded function defined by $f_{\mu}(x)=\int_{G} \int_{G} f(s x t) d \mu(t) d \mu(s)$ for all $x \in G$.

Note that if $\mu * \mu=\mu$, then $f$ is $\mu$-biinvariant if and only if $\int_{G} f(t x) d \mu(t)=$ $\int_{G} f(x t) d \mu(t)=f(x)$ for all $x \in G$.

Definition 1.1. A measure $\mu \in M(G)$ is a Gelfand measure if $\check{\bar{\mu}}=\mu * \mu=\mu$ and the Banach algebra $\mu * M(G) * \mu$ is commutative under the convolution. $\mu$ is a generalized Gelfand measure if $\mu * \mu=\mu$ and the Banach algebra $\mu * M(G) * \mu$ is commutative.

Any non-zero solution $\phi \in C_{b}(G)$ of the functional equation

$$
\int_{G} \phi(x t y) d \mu(t)=\phi(x) \phi(y) \text { for all } x, y \in G,
$$

is a $\mu$-spherical function.

$\mu$-spherical functions have been introduced and studied by Akkouchi and Bakali (see [1] and [2]).

When $H$ is a compact subgroup of $G$ and $d h$ is the normalized Haar measure of $H$, then $d h$ is a generalized Gelfand measure of $G$ if and only if $(G, H)$ is a Gelfand pair (see [7]). 
Finally, if $\mu \in M(G)$ is a generalized Gelfand measure, then, according to Definition 1.1,

$L_{1}^{\mu}(G)=\mu * L_{1}(G, d x) * \mu=\left\{f \in L_{1}(G, d x) \mid f=f * \mu=\mu * f=\mu * f * \mu=f^{\mu}\right\}$

is a commutative Banach algebra, where

$$
f^{\mu}(x)=\mu * f * \mu(x):=\int_{G} \int_{G} f\left(t^{-1} x s^{-1}\right) \Delta^{-1}(s) d \mu(t) d \mu(s),
$$

$\Delta$ being the modular function of $G$.

Furthermore, note that if $G$ is unimodular (i.e., $\Delta=1$ ), $\mu \in M(G)$ is $K$ invariant and $f \in L_{1}(G, d x)$, then we have

$$
\begin{aligned}
\left(M_{K}(f)\right)^{\mu}(x)= & \int_{G} \int_{G} M_{K}(f)\left(t^{-1} x s^{-1}\right) d \mu(t) d \mu(s) \\
= & \int_{K^{+}} \int_{G} \int_{G} f\left(k \cdot t^{-1} k \cdot x k \cdot s^{-1}\right) d k d \mu(t) d \mu(s) \\
& +\int_{K^{-}} \int_{G} \int_{G} f\left(k \cdot s^{-1} k \cdot x k \cdot t^{-1}\right) d k d \mu(t) d \mu(s) .
\end{aligned}
$$

Since $\check{\mu}$ is also $K$-invariant, we have

$$
\begin{array}{r}
\int_{K^{+}} \int_{G} \int_{G} f\left(k \cdot t^{-1} k \cdot x k \cdot s^{-1}\right) d k d \mu(t) d \mu(s) \\
=\int_{K^{+}} \int_{G} \int_{G} f\left(t^{-1} k \cdot x s^{-1}\right) d k d \mu(t) d \mu(s)
\end{array}
$$

and

$$
\begin{array}{r}
\int_{K^{-}} \int_{G} \int_{G} f\left(k \cdot s^{-1} k \cdot x k \cdot t^{-1}\right) d k d \mu(t) d \mu(s) \\
=\int_{K^{-}} \int_{G} \int_{G} f\left(t^{-1} k \cdot x s^{-1}\right) d k d \mu(t) d \mu(s),
\end{array}
$$

which implies that

$\left(M_{K}(f)\right)^{\mu}(x)=\int_{K} \int_{G} \int_{G} f\left(s^{-1} k \cdot x t^{-1}\right) d k d \mu(t) d \mu(s)=M_{K}\left(f^{\mu}\right)(x)$ for all $x \in G$.

\section{General Properties}

We fix a measure $\mu$ in $M(G)$. The following result gives necessary and sufficient conditions for a function to be a solution of equation (1.3) and explains why we restrict ourselves to continuous and bounded solutions. 
Theorem 2.1. Let $\mu \in M(G)$. Let $f$ be a measurable and essentially bounded function on $G$. Then the following conditions are equivalent

$$
\begin{aligned}
\int_{G}\left\{\int_{K} f(x t k \cdot y) d k\right\} d \mu(t) & =f(x) f(y) \text { for almost all } x, y \in G, \\
M_{K}(\check{\mu} * h * f) & =\langle h, \check{f}\rangle f \text { for all } h \in L_{1}(G, d x), \\
M_{K}(\check{\mu} * \nu * f) & =\langle\nu, \check{f}\rangle f \text { for all } \nu \in M(G), \\
M_{K}\left(\check{\mu} * \delta_{x} * f\right) & =\check{f}(x) f \text { for all } x \in G .
\end{aligned}
$$

Consequently, if $f$ satisfies one of these conditions, then it is continuous.

Proof. $(2.1) \Rightarrow(2.2)$. For all $g, h \in \mathcal{K}(G)$, we have

$$
\begin{aligned}
\langle h, \check{f}\rangle\langle f, g\rangle & =\int_{G} \int_{G} f\left(x^{-1}\right) f(y) h(x) g(y) d x d y \\
& =\int_{G} \int_{G} \int_{K} \int_{G} f\left(x^{-1} t k \cdot y\right) h(x) g(y) d x d y d k d \mu(t) \\
& =\int_{G} \int_{K} \int_{G}(h * f)\left(t^{-1} k \cdot y\right) g(y) d k d y d \check{\mu}(t) \\
& =\int_{G} \int_{K}(\check{\mu} * h * f)(k \cdot y) g(y) d k d y=\left\langle M_{K}(\check{\mu} * h * f), g\right\rangle .
\end{aligned}
$$

Consequently, $M_{K}(\check{\mu} * h * f)=\langle h, \check{f}\rangle f$, almost everywhere for all $h \in$ $L_{1}(G, d x)$.

Now by choosing $h \in \mathcal{K}(G)$ such that $\langle h, \check{f}\rangle \neq 0$ and by using ([6], 14.9.2), we deduce that $f$ is a continuous function and we get (2.2).

$(2.2) \Rightarrow(2.3)$. By $([6] 14.11 .1)$, there exists a sequence of regularizing functions $f_{n} \in L_{1}(G, d x)$.

In view of $(2.2)$, for all $h \in \mathcal{K}(G)$ we have

$$
\left\langle M_{K}\left(\check{\mu} * \nu * f_{n} * f\right), h\right\rangle=\left\langle\nu * f_{n}, \check{f}\right\rangle\langle f, h\rangle \text { for all } \nu \in M(G) \text { and } n \in \mathbb{N} \text {. }
$$

\section{Since}

$$
\left\langle M_{K}\left(\check{\mu} * \nu * f_{n} * f\right), h\right\rangle=\left\langle\check{\mu} * \nu * f_{n} * f, L_{K}(h)\right\rangle=\left\langle\check{\nu} * \mu * L_{K}(h), f_{n} * f\right\rangle,
$$

where $L_{K}(h)=\int_{K}(k \cdot h) \bmod (k) d k$ and $\bmod (k)$ is defined by the formula $\int_{G} g(k$. $x) d x=\bmod (k) \int_{G} g(x) d x$ for all $g \in L_{1}(G, d x)$ (cf. [6], 14.3.6.1.), by letting $n \longrightarrow+\infty$ and by using ([6], 14.11.1), we get

$$
\left\langle\check{\nu} * \mu * L_{K}(h), f\right\rangle=\langle\nu, \check{f}\rangle\langle f, h\rangle=\left\langle M_{K}(\check{\mu} * \nu * f), h\right\rangle .
$$

In view of $([6], 14.9 .2))$ and the fact that $f$ is continuous, we obtain (2.3).

$(2.4) \Rightarrow(2.1)$. Since $\left(\check{\mu} * \delta_{x} * f\right)(y)=\int_{G} f\left(x^{-1} t y\right) d \mu(t)$, we get for all $z \in G$

$$
M_{K}\left(\check{\mu} * \delta_{x} * f\right)(z)=\int_{K}\left(\check{\mu} * \delta_{x} * f\right)(k \cdot z) d k
$$




$$
=\int_{K} \int_{G} f\left(x^{-1} t k \cdot z\right) d k d \mu(t)=f\left(x^{-1}\right) f(z),
$$

which implies (2.1).

The following theorem explains some relations existing between solutions of equation (1.3) and continuous characters of the commutative Banach algebra $\mu * M_{K}\left(L_{1}(G, d x)\right) * \mu$, where $\mu$ is a generalized Gelfand measure which is $K$ invariant.

Theorem 2.2. Let $G$ be unimodular, let $\mu$ be a $K$-invariant generalized Gelfand measure and let $\omega \in C_{b}(G)$. Then the map

$$
f \longmapsto\langle f, \omega\rangle:=\int_{G} f(x) \omega(x) d x
$$

is a character of the commutative Banach algebra $\mu * M_{K}\left(L_{1}(G, d x)\right) * \mu$ if and only if $\omega$ is a nonzero solution of equation (1.3).

Proof. To prove Theorem 2.2, we need the following result.

Proposition 2.1. Let $\mu$ be a K-invariant generalized Gelfand measure. If $\omega \in C_{b}(G)$ is a solution of equation (1.3), then $\omega$ is a $\mu$-biinvariant function on $G$ and $\omega$ is $K$-invariant.

Consequently,

$$
\int_{G} \omega(x t y) d \mu(t)=\int_{G} \omega(y t x) d \mu(t) \quad \text { for all } x, y \in G .
$$

Proof. According to equation (1.3), we have

$$
\int_{K} \int_{G} \int_{G} \omega(x t s k \cdot y) d k d \mu(t) d \mu(s)=\int_{G} \omega(x t) d \mu(t) \omega(y) .
$$

Since $\mu * \mu=\mu$, we get

$$
\omega(y) \int_{G} \omega(x t) d \mu(t)=\omega(x) \omega(y) \text { for all } x, y \in G .
$$

which implies that

$$
\int_{G} \omega(x t) d \mu(t)=\omega(x), \quad x \in G
$$

On the other hand,

$$
\int_{K} \int_{G} \int_{G} \omega(x t k \cdot(s y)) d k d \mu(t) d \mu(s)=\omega(x) \int_{G} \omega(s y) d \mu(s) .
$$


Since $\mu * \mu=\mu, \mu$ is $K$-invariant and $\int_{G} \omega(x s) d \mu(s)=\omega(x)$, we get from

$$
\begin{gathered}
\int_{G} \int_{G} \int_{K} \omega(x t k \cdot(s y)) d k d \mu(t) d \mu(s) \\
=\int_{G} \int_{K^{+}} \int_{G} \omega(x t k \cdot s k \cdot y) d k d \mu(t) d \mu(s)+\int_{G} \int_{K^{-}} \int_{G} \omega(x t k \cdot y k \cdot s) d k d \mu(t) d \mu(s)
\end{gathered}
$$

and

$$
\begin{aligned}
& \int_{G} \int_{K^{+}} \int_{G} \omega(x t k \cdot s k \cdot y) d k d \mu(t) d \mu(s)=\int_{K^{+}} \int_{G} \omega(x t k \cdot y) d k d \mu(t), \\
& \int_{G} \int_{K^{-}} \int_{G} \omega(x t k \cdot y k \cdot s) d k d \mu(t) d \mu(s)=\iint_{G} \omega(x t k \cdot y) d k d \mu(t)
\end{aligned}
$$

that

$$
\int_{G} \int_{K} \omega(x t k \cdot y) d k d \mu(t)=\omega(x) \int_{G} \omega(s y) d \mu(s) \text { for all } x, y \in G .
$$

Thus

$$
\int_{G} \omega(s y) d \mu(s)=\omega(y) \text { for all } y \in G .
$$

Now, since $\mu$ is a generalized Gelfand measure, by using ([9], Proposition 2.1) we obtain

$$
\int_{G} \omega(x s y) d \mu(s)=\int_{G} \omega(y s x) d \mu(s), \quad x, y \in G .
$$

If we fix $k^{\prime} \in K$, then using equation (1.3), we get

$$
\int_{K} \int_{G} \omega\left(x t k \cdot\left(k^{\prime} \cdot y\right)\right) d k d \mu(t)=\omega(x) \omega\left(k^{\prime} \cdot y\right) .
$$

So from the fact that $K$ is compact and hence unimodular it follows that the first term of equation (2.5) becomes

$$
\int_{K} \int_{G} \omega\left(x t k \cdot\left(k^{\prime} \cdot y\right)\right) d k d \mu(t)=\int_{K} \int_{G} \omega(x t k \cdot y) d k d \mu(t)
$$

whence we deduce that $\omega\left(k^{\prime} \cdot x\right)=\omega(x)$ for all $x \in G$. The proof of the proposition is finished.

Proof of Theorem 2.2. Let $\omega \in C_{b}(G)$ be a solution of equation (1.3), then for all $f, g \in L_{1}(G, d x)$, we have

$$
\left\langle\int_{K}(k \cdot f)^{\mu} d k * \int_{K}\left(k^{\prime} \cdot g\right)^{\mu} d k^{\prime}, \omega\right\rangle=\int_{K} \int_{K}\left\langle(k \cdot f)^{\mu} *\left(k^{\prime} \cdot g\right)^{\mu}, \omega\right\rangle d k d k^{\prime}
$$




$$
\begin{aligned}
= & \int_{K} \int_{K} \int_{G} \int_{G}(k \cdot f)^{\mu}(y)\left(k^{\prime} \cdot g\right)^{\mu}\left(y^{-1} x\right) \omega(x) d k d k^{\prime} d x d y \\
= & \int_{K} \int_{K} \int_{G} \cdots \int_{G}(k \cdot f)\left(t^{-1} y s^{-1}\right)\left(k^{\prime} \cdot g\right)\left(l^{-1} y^{-1} x r^{-1}\right) \\
& \times \int_{K} \int_{K} \int_{G} \cdots \int_{G} \int_{G}(k \cdot f)(y)\left(k^{\prime} \cdot g\right)(x) \\
& \times \omega(\text { tylsxr }) d x d y d k d k^{\prime} d x d y d \mu(t) d \mu(s) d \mu(l) d \mu(r)
\end{aligned}
$$

Hence by Proposition 2.1 and $\mu * \mu=\mu$,

$$
\begin{aligned}
\int_{K} \int_{K} \int_{G} & \cdots \int_{G} \int_{G}(k \cdot f)(y)\left(k^{\prime} \cdot g\right)(x) \\
& \times \omega(t y l s x r) d k d k^{\prime} d x d y d \mu(l) d \mu(t) d \mu(s) d \mu(r) \\
= & \int_{K} \int_{K} \int_{G} \int_{G} \int_{G}(k \cdot f)(y)\left(k^{\prime} \cdot g\right)(x) \omega(y s x) d x d y d k d k^{\prime} d \mu(s) \\
= & \int_{K} \int_{K} \int_{G} \int_{G} \int_{G}(k \cdot f)(y)\left(k^{\prime} \cdot g\right)(x) k^{-1} \cdot \omega\left(k^{-1} \cdot y s k^{-1} \cdot x\right) d x d y d k d k^{\prime} d \mu(s) \\
= & \int_{K} \int_{K} \int_{G} \int_{G} \int_{G} f(k \cdot y)\left(k^{\prime} \cdot g\right)(x) \omega(k \cdot y s k \cdot x) d x d y d k d k^{\prime} d \mu(s) \\
= & \int_{K} \int_{K} \int_{G} \int_{G} \int_{G} f(y)\left(k^{\prime} \cdot g\right)(x) \omega(y s k \cdot x) d x d y d k d k^{\prime} d \mu(s),
\end{aligned}
$$

which follows from $\int_{G} k \cdot f(x) d x=\int_{G} f(x) d x$ for all $f \in L_{1}(G, d x)$ and $k \in K$. Consequently, we have

$$
\begin{aligned}
\left\langle\int_{K}(k \cdot f)^{\mu} d k * \int_{K}\left(k^{\prime} \cdot g\right)^{\mu} d k^{\prime}, \omega\right\rangle \\
=\int_{K} \int_{K} \int_{G} \int_{G} \int_{G} f(y)\left(k^{\prime} \cdot g\right)(x) \omega(y s k \cdot x) d x d y d k d k^{\prime} d \mu(s) \\
=\int_{K} \int_{G} \int_{G}\left(k^{\prime} \cdot g\right)(x) f(y) \omega(y) \omega(x) d x d y d k^{\prime} \\
=\int_{K} \int_{K} \int_{G} \int_{G}(k \cdot f)^{\mu}(y)\left(k^{\prime} \cdot g\right)^{\mu}(x) \omega(y) \omega(x) d x d y d k d k^{\prime} \\
=\left\langle\int_{K}(k \cdot f)^{\mu} d k, \omega\right\rangle\left\langle\int_{K}\left(k^{\prime} \cdot g\right)^{\mu} d k^{\prime}, \omega\right\rangle,
\end{aligned}
$$


which means that $f \longmapsto\langle f, \omega\rangle$ is a character of $\mu *\left(M_{K}\left(L_{1}(G, d x)\right)\right) * \mu$.

Let conversely $\chi$ be a continuous character of the commutative Banach algebra $\mu *\left(M_{K}\left(L_{1}(G, d x)\right)\right) * \mu$. Then $f \longmapsto \chi\left(\left(M_{K}(f)\right)^{\mu}\right)$ is a continuous linear mapping of the Banach algebra $L_{1}(G, d x)$ into $\mathbb{C}$. Consequently, there exists $\omega \in L_{\infty}(G)$ such that

$$
\chi\left(\left(M_{K}(f)\right)^{\mu}\right)=\langle f, \omega\rangle \text { for all } f \in L_{1}(G, d x) .
$$

In addition, $\omega$ may be chosen continuous: Let $f_{0} \in \mathcal{K}(G)$ be a $K$-invariant function such that $\chi\left(\left(M_{K}\left(f_{0}\right)\right)^{\mu}\right)=1$. Then for all $f \in \mathcal{K}(G)$ we have

$$
\begin{aligned}
\langle f, \omega\rangle & \left.=\chi\left(\left(M_{K}\left(f_{0}\right)\right)^{\mu}\right) \chi\left(\left(M_{K}(f)\right)^{\mu}\right)=\chi\left(\left(M_{K}(f)\right)^{\mu}\right) *\left(M_{K}\left(f_{0}\right)\right)^{\mu}\right) \\
& =\chi\left(\left(M_{K}\left(M_{K}(f) * f_{0}^{\mu}\right)^{\mu}\right)=\left\langle M_{K}(f) * f_{0}^{\mu}, \omega\right\rangle=\left\langle M_{K}(f), \omega *\left(f_{0}^{\mu}\right)^{\vee}\right)\right\rangle \\
& =\left\langle f, M_{K}\left(\omega *\left(f_{0}^{\mu}\right)^{\vee}\right)\right\rangle .
\end{aligned}
$$

Consequently, $\omega=M_{K}\left(\omega *\left(f_{0}^{\mu}\right)^{\vee}\right)$ locally almost everywhere.

By using $L_{\infty}(G, d x) * L_{1}(G, d x) \subseteq C_{b}(G)$, we conclude that $\omega$ is a continuous function on $G$.

On the other hand, in view of

$$
\chi\left(\left(M_{K}(f)\right)^{\mu}\right)=\chi\left(\left(M_{K}\left(f^{\mu}\right)\right)^{\mu}\right)=\left\langle f^{\mu}, \omega\right\rangle=\left\langle f, \omega_{\mu}\right\rangle,
$$

we deduce that $\langle f, \omega\rangle=\left\langle f, \omega_{\mu}\right\rangle$ for all $f \in L_{1}(G, d x)$, which implies that $\omega_{\mu}=\omega$, i.e., $\omega$ is $\mu$-biinvariant.

To show that $\omega$ is a $K$-invariant function, we will use the formula $M_{K}\left(M_{K}(f)\right)$ $=M_{K}(f) ; f \in L_{1}(G, d x)$. For all $f \in L_{1}(G, d x)$ we have

$$
\langle f, \omega\rangle=\chi\left(\left(M_{K}(f)\right)^{\mu}\right)=\chi\left(\left(M_{K}\left(M_{K}(f)\right)\right)^{\mu}\right)=\left\langle M_{K}(f), \omega\right\rangle=\left\langle f, M_{K}(\omega)\right\rangle,
$$

from which we conclude that $\omega=M_{K}(\omega)$ and $k^{\prime} \cdot \omega=\omega$ for all $k^{\prime} \in K$.

Now we are going to show that $\omega$ is a solution of equation (1.3).

For all $f, g \in L_{1}(G, d x)$ we have

$$
\begin{aligned}
\left\langle\left(\int_{K}(k \cdot f) d k\right)^{\mu} *\left(\int_{K}\left(k^{\prime} \cdot g\right) d k^{\prime}\right)^{\mu}, \omega\right\rangle \\
=\int_{G} \int_{G} \int_{K} \int_{G} \int_{K} f(y) k^{\prime} \cdot g(x) \omega(k \cdot y t x) d k d k^{\prime} d x d y d \mu(t) \\
=\int_{G} \int_{G} \int_{K} \int_{K} \int_{G} f(y) g(x) \omega\left(k^{\prime} \cdot y t k \cdot x\right) d k d k^{\prime} d x d y d \mu(t) \\
=\int_{G} \int_{G} \int_{K} \int_{K^{+}} \int_{G} f(y) g(x) k^{\prime-1} \cdot \omega\left(y t\left(k^{\prime-1} k\right) \cdot x\right) d \mu(t) d k d k^{\prime} d x d y \\
\quad+\int_{G} \int_{G} \int_{K} \int_{K^{-}} \int_{G} f(y) g(x) k^{\prime-1} \cdot \omega\left(\left(k^{\prime-1} k\right) \cdot x t y\right) d k d k^{\prime} d x d y d \mu(t) \\
=\int_{G} \int_{G} \int_{K} \int_{K} \int_{G} f(y) g(x) \omega\left(y t\left(k^{\prime-1} k\right) \cdot x\right) d k d k^{\prime} d x d y d \mu(t)
\end{aligned}
$$




$$
=\iint_{G} \int_{G} \int_{G} f(y) g(x) \omega(y t k \cdot x) d k d x d y d \mu(t)
$$

and it follows that

$$
\int_{G} \int_{G} \int_{K} \int_{G} f(y) g(x) \omega(y t k \cdot x) d k d x d y d \mu(t)=\int_{G} \int_{G} f(y) g(x) \omega(y) \omega(x) d x d y
$$

for all $f, g \in L_{1}(G, d x)$. Consequently, $\omega$ is a solution of equation (1.3).

On the other hand, in view of $\chi(f)=\langle f, \omega\rangle$ for all $f \in \mu * M_{K}\left(L_{1}(G, d x)\right) * \mu$ we deduce that $\omega \neq 0$. This ends the proof of Theorem 2.2.

\section{Solutions of Equation (1.3)}

In this section, $\mu$ is a generalized Gelfand measure which is $K$-invariant and we assume that every closed ideal of the commutative Banach algebra $L_{1}^{\mu}(G)$ is contained in some maximal regular ideal. The latter condition is satisfied for example in the following situations:

- $G$ is a compact group and $\mu$ is a Gelfand measure (cf. [2]).

- $G$ is unimodular with a growth being almost of polynomial type and $\mu$ is a Gelfand measure with compact support (cf. [10], Theorem 3.3.5, [13], Corollary on p. 227, and [8]).

In Theorem 3.1 below, we give a full description of continuous and bounded solutions of the equation

$$
\int_{G}\left\{\int_{K} f(x t k \cdot y) d k\right\} d \mu(t)=f(x) f(y), \quad x, y \in G .
$$

Theorem 3.1. Let $\mu$ be a Gelfand measure which is K-invariant. Let $f$ be a nonzero continuous and bounded solution of the functional equation (3.1). Then there exists a $\mu$-spherical function $\psi$ on $G$ such that

$$
f(x)=\int_{K} \psi(k \cdot x) d k, \quad x \in G .
$$

Conversely, any function of this form is a solution of equation (3.1).

Proof. Let $\psi$ be a $\mu$-spherical function. Let $f$ be a continuous and bounded function defined by the formula

$$
f(x)=\int_{K} \psi(k \cdot x) d k, \quad x \in G .
$$

Hence $f\left(x t k^{\prime} \cdot y\right)=\int_{K} \psi\left(k \cdot\left(x t k^{\prime} \cdot y\right)\right) d k$ and

$$
\begin{aligned}
& \int_{G} \int_{K} f\left(x t k^{\prime} \cdot y\right) d k^{\prime} d \mu(t)=\int_{K} \int_{K} \int_{G} \psi\left(k \cdot\left(x t k^{\prime} \cdot y\right)\right) d k d k^{\prime} d \mu(t) \\
& =\int_{K^{+}} \int_{K} \int_{G} \psi\left(k \cdot x t\left(k k^{\prime}\right) \cdot y\right) d k d k^{\prime} d \mu(t)+\int_{K^{-}} \int_{K} \int_{G} \psi\left(\left(k k^{\prime}\right) \cdot y t k \cdot x\right) d k d k^{\prime} d \mu(t)
\end{aligned}
$$




$$
\begin{aligned}
& =\int_{K} \int_{K} \int_{G} \psi\left(\left(k k^{\prime}\right) \cdot y t k \cdot x\right) d k d k^{\prime} d \mu(t) \\
& =\int_{K} \int_{K} \psi\left(\left(k k^{\prime}\right) \cdot y\right) \psi(k \cdot x) d k d k^{\prime}=\int_{K} \psi\left(k^{\prime} \cdot y\right) d k^{\prime} \int_{K} \psi(k \cdot x) d k=f(x) f(y) .
\end{aligned}
$$

Conversely, if $f \in C_{b}(G)$ is a non-zero continuous and bounded solution of the functional equation (3.1), then it has the form (3.2). To prove this first we choose an arbitrary function $g \in L_{1}(G, d x)$.

Multiplying (3.1) by $g(x)$ and integrating the result over $G$, we get

$$
\int_{G} g(x)\left(\int_{K} \int_{G} f(x t k \cdot y) d k d \mu(t)\right) d x=f(y) \int_{G} f(x) g(x) d x \text { for all } y \in G \text {. }
$$

Hence, in view of

$$
\int_{K} \int_{G} f(x t k \cdot y) d k d \mu(t)=\int_{K} \int_{G} f(k \cdot y t x) d k d \mu(t)=\int_{K}\left(\check{\mu}_{k \cdot y} * f\right)(x) d k,
$$

we get

$$
\int_{G} f(x)\left[\int_{K}\left(\left(\check{\nu_{k \cdot y}}\right) * g\right)(x) d k-f(y) g(x)\right] d x=0 \text { for all } y \in G,
$$

where $\nu=\check{\mu}$.

We shall now consider the sets $A=\left\{\int_{K}\left(\left(\check{\nu_{k \cdot y}}\right) * g\right) d k-f(y) g \mid g \in L_{1}(G, d x)\right.$, $y \in G\}$ and $B=\left\{h^{\mu}, h \in A\right\}$.

To establish that $I=\operatorname{Lin}(B)$ is an ideal in the algebra $L_{1}^{\mu}(G, d x)$, suppose that $h^{\mu} \in B$, where $h=\int_{K}\left(\left(\nu_{k \cdot y}\right) * g\right) d k-f(y) g \mid y \in G, g \in L_{1}(G, d x)$.

Now for all $L \in L_{1}^{\mu}(G, d x)$ we have

$$
L * h^{\mu}=(L * h)^{\mu}=\left(\int_{K}\left(\left(\nu_{k} \cdot y\right) * N\right) d k-f(y) N\right)^{\mu},
$$

where $N=L * g$, which implies that $L * h^{\mu} \in I$, since $I$ is a linear subspace of $L_{1}^{\mu}(G, d x)$. Thus we conclude that $I$ is an ideal of the Banach algebra $L_{1}^{\mu}(G, d x)$.

There are two cases:

Case (1). $I=L_{1}^{\mu}(G, d x)$. Hence for all $h \in L_{1}(G, d x)$,

$$
\int_{G} f(x) h^{\mu} d x=\int_{G} f(x) h(x)=0,
$$

which implies that $f(x)=0$ for all $x \in G$, which contradicts our assumption.

Case (2). $I$ is a proper ideal of the Banach algebra $L_{1}^{\mu}(G, d x)$.

By the assumption of the theorem, there exists a regular maximal ideal $I_{\max }$ of $L_{1}^{\mu}(G, d x)$, which contains $I$.

Now by adapting the same method as used in ([13], Theorem 1, p. 412) we conclude that there exists a continuous character $\chi_{0}$ of the Banach algebra 
$L_{1}^{\mu}(G, d x)$ such that $\chi_{0}(\theta)=0$ for all $\theta \in I_{\max }$. Hence by [1], there exists a $\mu$-spherical function $\psi$ such that

$$
\int_{G} h^{\mu}(x) \psi(x) d x=0 \text { for all } h^{\mu} \in I
$$

Therefore

$$
\int_{G} \int_{K}\left(\left(\tilde{\nu_{k} \cdot y}\right) * g\right)(x) \psi(x) d x d k=f(y) \int_{G} \psi(x) g(x) d x
$$

for all $y \in G$ and for all $g \in L_{1}(G, d x)$.

Since

$$
\int_{G} \int_{K}((\check{\nu} \cdot y) * g)(x) \psi(x) d x d k=\int_{K} \psi(k \cdot y) d k \int_{G} g(x) \psi(x) d x,
$$

then by choosing $g_{0} \in L_{1}(G, d x)$ such that $\int_{G} g_{0}(x) \psi(x) d x \neq 0$ we get

$$
f(y)=\int_{K} \psi(k \cdot y) d k \text { for all } y \in G .
$$

The proof of the theorem is thus completed.

\section{Badora's Equation in Lie Groups}

In this section, we characterize solutions of the equation

$$
\int_{G}\left\{\int_{K} f(x t k \cdot y) d k\right\} d \mu(t)=f(x) f(y), \quad x, y \in G,
$$

on a connected Lie group $G$ as joint eigenfunctions of certain operators associated to the left invariant differential operators, where $K$ is a compact subgroup of the group $\operatorname{Aut}(G)$ of all mappings of $G$ onto $G$ that are simultaneously automorphisms and homeomorphisms.

This extends the previous results obtained by Stetkær in [17] for equation (1.4) and by the authors in [3] for the equation

$$
\int_{G} f(x t y) d \mu(t)+\int_{G} f(x t \sigma(y)) d \mu(t)=2 f(x) f(y), \quad x, y \in G,
$$

where $\sigma$ is a continuous automorphism of $G$ such that $\sigma \circ \sigma=I$.

In the sequel, we need the following notations.

Let $G$ be a connected Lie group. $\mathbb{D}(G)$ denote the algebra of the left invariant differential operators on $G$, i.e., for all $D \in \mathbb{D}(G)$ and for all $a \in G$

$$
L(a) D f=D L(a) f \text { for all } f \in C^{\infty}(G),
$$

where $L(a) f(x)=f\left(a^{-1} x\right)$ for all $x \in G$.

We recall (see [17], Proposition II.3) that $K$ has a Lie group structure, the canonical map $K \times G \rightarrow G$ sending $(k, x)$ onto $k \cdot x$ is $C^{\infty}$ and if $f \in C^{\infty}(G)$, 
then so does $k \cdot f$ for any $k \in K$ because continuous homomorphisms between Lie groups automatically are $C^{\infty}$.

Throughout this section, we assume that $\mu$ satisfies the following conditions:

1) $\mu$ is a $K$-invariant measure with compact support on $G$ and

2) $\mu * \mu=\mu$.

The symbol $C_{\mu}^{\infty}(G)=\check{\mu} * C^{\infty} * \Delta \check{\mu}$ stands for all $C^{\infty}$-functions which are $\mu$-biinvariant on $G$. The subspace of $C_{\mu}^{\infty}(G)$ of functions which are $K$-invariant will be denoted by $C_{\mu, K}^{\infty}(G)$.

For any operator $D$ on $C^{\infty}(G)$, we define the new operator by

$$
D_{\mu}^{K} f(x)=D\left\{M_{K}\left(L\left(x^{-1}\right) f\right)_{\mu}\right\}(e)
$$

for all $f \in C^{\infty}(G)$ and $x \in G$.

We will next describe some properties of $D_{\mu}^{K}$ that will be used later.

Proposition 4.1. Our assumptions imply

(1) $D_{\mu}^{K}$ is a left invariant operator;

(2) $k \cdot D_{\mu}^{K} f=D_{\mu}^{K} k \cdot f$ for all $k \in K$ and for all $f \in C^{\infty}(G)$;

(3) $\left(D_{\mu}^{K} f\right)(e)=D\left(M_{K} f_{\mu}\right)(e)$. In particular, if $f$ is a $\mu$-biinvariant and $K$ invariant function on $G$, then we have $\left(D_{\mu}^{K} f\right)(e)=(D f)(e)$;

(4) If $f$ is a solution of equation (4.1) which satisfies $\int_{G} f(x t) d \mu(t)=f(x)$ for all $x \in G$, then $f$ is an eigenfunction of the operators $D_{\mu}^{K}$. More precisely $D_{\mu}^{K} f=(D f)(e) f$ and, consequently, $f$ is analytic on $G$.

Proof. (1) First we choose an arbitrary function $f \in C^{\infty}(G)$. For each fixed $a \in G$ and for all $x \in G$, we have

$$
\begin{gathered}
L(a)\left(D_{\mu}^{K} f\right)(x)=D_{\mu}^{K} f\left(a^{-1} x\right)=D\left\{M_{K}\left(L\left(x^{-1} a\right) f\right)_{\mu}\right\}(e) \\
=D\left\{M_{K}\left(L\left(x^{-1}\right) L(a) f\right)_{\mu}\right\}(e)=D_{\mu}^{K}(L(a) f)(x),
\end{gathered}
$$

which implies that $D_{\mu}^{K}$ is a left invariant operator on $G$.

(2) If we now take arbitrary $k^{\prime} \in K$, then for all $x \in G$ we have

$$
k^{\prime-1} \cdot\left(D_{\mu}^{K} f\right)(x)=\left(D_{\mu}^{K} f\right)\left(k^{\prime} \cdot x\right)=D\left\{M_{K}\left(L\left(k^{\prime} \cdot x^{-1}\right) f\right)_{\mu}\right\} .
$$

For all $y \in G$ we have

$$
\begin{aligned}
M_{K} & \left\{L\left(k^{\prime} \cdot x^{-1} f\right)_{\mu}\right\}(y)=\int_{K}\left(L\left(k^{\prime} \cdot x^{-1}\right) f\right)_{\mu}(k \cdot y) d k \\
& =\int_{G} \int_{K} \int_{G} L\left(k^{\prime} \cdot x^{-1}\right) f(t k \cdot y s) d \mu(t) d \mu(s) d k \\
& =\int_{G} \int_{G} \int_{K} f\left(k^{\prime} \cdot x t k \cdot y s\right) d \mu(t) d \mu(s) d k
\end{aligned}
$$

and

$$
M_{K}\left\{\left(L\left(x^{-1}\right) k^{\prime-1} \cdot f\right)_{\mu}\right\}(y)=\int_{K}\left(L\left(x^{-1}\right) k^{\prime-1} \cdot f\right)_{\mu}(k \cdot y) d k
$$




$$
\begin{aligned}
& =\int_{G} \int_{K} \int_{G} L\left(x^{-1}\right) k^{\prime-1} \cdot f(t k \cdot y s) d k d \mu(t) d \mu(s) \\
& =\int_{G} \int_{K} \int_{G} k^{\prime-1} \cdot f(x t k \cdot y s) d k d \mu(t) d \mu(s) \\
& =\int_{G} \int_{K} \int_{G} f\left(k^{\prime} \cdot x k^{\prime} \cdot t\left(k^{\prime} k\right) \cdot y k^{\prime} \cdot s\right) d k d \mu(t) d \mu(s) .
\end{aligned}
$$

Since $\mu$ is $K$-invariant and $K$ is unimodular, we get

$$
\begin{gathered}
\int_{G} \int_{K} \int_{G} f\left(k^{\prime} \cdot x k^{\prime} \cdot t\left(k^{\prime} k\right) \cdot y k^{\prime} \cdot s\right) d k d \mu(t) d \mu(s) \\
=\int_{G} \int_{K} \int_{G} f\left(k^{\prime} \cdot x t k \cdot y s\right) d k d \mu(t) d \mu(s) .
\end{gathered}
$$

Consequently, we have the equality

$$
k \cdot D_{\mu}^{K} f=D_{\mu}^{K} k \cdot f \text { for all } k \in K .
$$

(4) Since $f$ is a solution of equation (4.1), then for all $x, y \in G$,

$$
\begin{aligned}
M_{K}\left(L\left(x^{-1}\right) f\right)_{\mu}(y) & =\int_{K}\left(L\left(x^{-1}\right) f\right)_{\mu}(k \cdot y) d k \\
& =\int_{G} \int_{G} \int_{K}\left(L\left(x^{-1}\right) f\right)(t k \cdot y s) d k d \mu(t) d \mu(s) \\
& =\int_{K} \int_{G} \int_{G} f(x t k \cdot y s) d k d \mu(t) d \mu(s) .
\end{aligned}
$$

In view of $\int_{G} f(x s) d \mu(s)=f(x)$ for all $x \in G$ we get

$$
M_{K}\left(L\left(x^{-1}\right) f\right)_{\mu}(y)=\int_{K} \int_{G} f(x t k \cdot y) d k d \mu(t)=f(x) f(y),
$$

which implies that $M_{K}\left(L\left(x^{-1}\right) f\right)_{\mu}=f(x) f$ and consequently $D_{\mu}^{K} f=(D f)(e) f$.

Now let us prove that any solution $f$ of (4.1) is analytic on $G$. We first show that $f \in C^{\infty}(G)$. Formula (2.2) shows that

$$
M_{K}(\check{\mu} * h * f)=\langle h, \check{f}\rangle f \text { for all } h \in L_{1}(G, d x) .
$$

Consequently, by choosing $h \in C_{\mu, K}^{\infty}(G)$ with compact support and such that $\langle h, \check{f}\rangle=1$, we get $h * f=M_{K}(\check{\mu} * h * f)=\langle h, \check{f}\rangle f=f$, i.e., $h * f=f$. Since $h \in C^{\infty}(G)$, so is the convolution and we conclude that $f \in C^{\infty}(G)$.

On the other hand, if $D=\triangle$ denotes the Laplace-Beltrami operator on $G$, then in view of $([11]$, p. 400$), \triangle$ is elliptic and has analytic coefficients. Since $\triangle f=\triangle_{\mu}^{K} f=\triangle(f)(e) f$, by using Theorem of S. Bernstein [12] we get that $f$ is analytic. This ends the proof of the proposition. 
We now state and prove the following proposition.

Proposition 4.2. With the assumptions above on $G, K$ and $\mu$ and if $D \in$ $\mathbb{D}(G)$, then for all $f \in C_{\mu, K}^{\infty}(G)$,

$$
D_{\mu}^{K} f=M_{K}(D f * \triangle \check{\mu})
$$

In particular the restriction of $D_{\mu}^{K}$ to $C_{\mu, K}^{\infty}(G)$ is an endomorphism.

Proof. Let $D \in \mathbb{D}(G)$ and $f \in C_{\mu, K}^{\infty}(G)$, then for all $x, y \in G$ we have

$$
\begin{gathered}
M_{K}\left(L\left(x^{-1}\right) f\right)_{\mu}(y)=\int_{G} \int_{G} \int_{K} f(x t k \cdot y s) d k d \mu(t) d \mu(s) \\
=\int_{G} \int_{K} f(x t k \cdot y) d \mu(t) d k=\int_{K} \int_{G} k^{-1} \cdot f\left(k^{-1} \cdot x k^{-1} \cdot t y\right) d \mu(t) d k .
\end{gathered}
$$

Since $\mu$ and $f$ are $K$-invariant, we get

$$
\begin{aligned}
& \int_{K} \int_{G} k^{-1} \cdot f\left(k^{-1} \cdot x k^{-1} \cdot t y\right) d \mu(t) d k=\int_{K} \int_{G} f\left(k^{-1} \cdot x t y\right) d \mu(t) d k \\
& =\int_{K} \int_{G} f(k \cdot x t y) d \mu(t) d k=\int_{K} \int_{G} L\left((k \cdot x t)^{-1}\right) f(y) d \mu(t) d k .
\end{aligned}
$$

Consequently,

$$
\begin{gathered}
D\left\{M_{K}\left(L\left(x^{-1}\right) f\right)_{\mu}\right\}(e)=\int_{K} \int_{G} L\left((k \cdot x t)^{-1}\right) D f(e) d k d \mu(t) \\
=\int_{K} \int_{G} D f(k \cdot x t) d k d \mu(t)=\int_{K} D f * \triangle \check{\mu}(k \cdot x) d k=M_{K}(D f * \triangle \check{\mu})(x),
\end{gathered}
$$

which proves that

$$
D_{\mu}^{K} f=M_{K}(D f * \triangle \check{\mu})
$$

From this it follows that $D_{\mu}^{K} f$ is a $K$-invariant function on $G$.

Now we are able to prove that $D_{\mu}^{K} f$ is a $\mu$-biinvariant function.

First, we show that $D f * \triangle \check{\mu}$ is $\mu$-biinvariant.

$$
\check{\mu} *(D f * \triangle \check{\mu}) * \triangle \check{\mu}=\check{\mu} * D f * \triangle \check{\mu}=D(\check{\mu} * f) * \triangle \check{\mu}=D f * \triangle \check{\mu},
$$

which proves that $D f * \triangle \check{\mu}$ is a $\mu$-biinvariant function on $G$.

Now for all $x \in G$ we have

$$
\begin{gathered}
\left(\check{\mu} * M_{K}(D f * \triangle \check{\mu}) * \triangle \check{\mu}\right)(x)=\int_{G} \int_{G} M_{K}(D f * \triangle \check{\mu})(t x s) d \mu(t) d \mu(s) \\
\left.=\int_{G} \int_{G} \int_{K}(D f * \triangle \check{\mu})(k \cdot t k \cdot x k \cdot s)\right) d k d \mu(t) d \mu(s)
\end{gathered}
$$




$$
\left.=\int_{G} \int_{G} \int_{K}(D f * \triangle \check{\mu})(t k \cdot x s)\right) d k d \mu(t) d \mu(s) .
$$

Since $D f * \triangle \check{\mu}$ is $\mu$-biinvariant, we get

$$
\begin{gathered}
\int_{G} \int_{G} \int_{K}(D f * \Delta \check{\mu})(t k \cdot x s) d \mu(t) d \mu(s) d k=\int_{K}(D f * \Delta \check{\mu})(k \cdot x) d k \\
=M_{K}(D f * \triangle \check{\mu})(x),
\end{gathered}
$$

from which we deduce that $D_{\mu}^{K} f$ is $\mu$-biinvariant.

In the next theorem, we prove the main result of this section.

Theorem 4.1. Let $\mu \in M(G)$ be a $K$-invariant, idempotent measure with compact support. If $f \in C(G)$, then the following statements are equivalent:

(1) $f$ is a solution of the equation

$$
\int_{G}\left\{\int_{K} f(x t k \cdot y) d k\right\} d \mu(t)=f(x) f(y) \text { for all } x, y \in G,
$$

(2) (a) $f$ is $\mu$-biinvariant,

(b) $f$ is $K$-invariant,

(c) $f \in C^{\infty}(G)$,

(d) $f$ is analytic,

(e) $f$ is a joint eigenfunction of the operators $D_{\mu}^{K}$ for all $D \in \mathbb{D}(G)$.

Proof. (1) $\Rightarrow(2)$ follows directly from Proposition 4.1. Conversely, suppose that the assumptions $(a),(b),(c), d$ and $(e)$ hold and let us consider the function defined on $G$ by

$$
F(y)=\int_{K} \int_{G} f(k \cdot x t y) d k d \mu(t)
$$

where $x$ is an arbitrary element of $G$.

It is easy to verify that $F$ is a $\mu$-biinvariant function on $G$. Concerning the $K$-invariance of $F$, for each fixed $k^{\prime} \in K$ and for all $y \in G$ we have

$F\left(k^{\prime} \cdot y\right)=\int_{G} \int_{K} f\left(k \cdot x t k^{\prime} \cdot y\right) d k d \mu(t)=\int_{G} \int_{K} k^{\prime-1} \cdot f\left(\left(k^{\prime-1} k\right) \cdot x k^{\prime-1} \cdot t y\right) d k d \mu(t)$.

Since $\mu$ and $f$ are $K$-invariant, we obtain

$$
\int_{G} \int_{K} k^{\prime-1} \cdot f\left(\left(k^{\prime-1} k\right) \cdot x k^{\prime-1} \cdot t y\right) d k d \mu(t)=\int_{G} \int_{K} f\left(\left(k^{\prime-1} k\right) \cdot x t y\right) d k d \mu(t),
$$

and from the left-invariance of $d k$ follows the equality

$$
\int_{G} \int_{K} f\left(\left(k^{\prime-1} k\right) \cdot x t y\right) d k d \mu(t)=\int_{G} \int_{K} f(k \cdot x t y) d k d \mu(t)=F(y),
$$

which proves that $F$ is $K$-invariant. 
Now $F(y)$ can be written in the form

$$
F(y)=\int_{G} \int_{K} L\left((k \cdot x t)^{-1}\right) f(y) d k d \mu(t) .
$$

By Proposition 4.1 $D_{\mu}^{K}$ is left invariant so that we get

$$
\begin{aligned}
D_{\mu}^{K} F(y) & =\int_{G} \int_{K} D_{\mu}^{K} f(k \cdot x t y) d k d \mu(t) \\
& =D(f)(e) \int_{G} \int_{K} f(k \cdot x t y) d k d \mu(t)=D(f)(e) F(y),
\end{aligned}
$$

which implies that

$$
D_{\mu}^{K} F(e)=D(f)(e) f(x) .
$$

In view of Proposition 4.1, $D_{\mu}^{K} F(e)=D F(e)$, and from (4.2) we get

$$
D(F-F(e) f)(e)=0 \text { for all } D \in \mathbb{D}(G) \text {. }
$$

Since $F-F(e) f$ is analytic on the connected Lie group $G$, by Helgason [11], we obtain $F=f(x) f$, which can be rewritten as

$$
\int_{G}\left\{\int_{K} f(k \cdot x t y) d k\right\} d \mu(t)=f(x) f(y) \text { for all } x, y \in G .
$$

By using the same methods as in the proof of Proposition 4.2 we get

$$
\int_{G}\left\{\int_{K} f(x t k \cdot y) d k\right\} d \mu(t)=\int_{G}\left\{\int_{K} f(k \cdot x t y) d k\right\} d \mu(t)=f(x) f(y)
$$

for all $x, y \in G$. This ends the proof of the theorem.

\section{ACKNOWLEDGEMENT}

We would like to express our thanks to the referee, without his helpful comments and valuable remarks our work could not have been done.

\section{REFERENCES}

1. M. Akkouchi and A. Bakali, Une généralisation des paires de Gelfand. Boll. Un. Mat. Ital. B (7) 6(1992), No. 4, 795-822.

2. M. Akкоuchi, Mesures de Gelfand et fonctions sphériques généralisées, Doctorat d'État. Uni. Ibn Tofail, Kenitra (Maroc), 1994.

3. M. Akkouchi, A. Bakali, B. Bouikhalene, and E. Elqorachi, On generalized d'Alembert functional equation. (submitted)

4. R. BADORA, On a joint generalization of Cauchy's and d'Alembert's functional equations. Aequationes Math. 43(1992), No. 1, 72-89.

5. W. Chojnacki, On some functional equation generalizing Cauchy's and d'Alembert's functional equations. Colloq. Math. 55(1988), No. 1, 169-178.

6. J. Dieudonné, Éléments d'analyse. Tome II: Chapitres XII à XV. Cahiers Scientifiques, Fasc. XXXI. Gauthier-Villars, Éditeur, Paris, 1968. 
7. J. Dieudonné, Eléments d'analyse. Tome VI. Cahiers Scientifiques, Fasc. XXXIX. Gauthier-Villars Éditeur, Paris, 1975.

8. E. ElqORAChi, Éléments de Gel'fand dans une algèbre de Banach involutive et certaines questions d'analyse harmonique. Doctorat de Troisième cycle. Uni. Cadi Ayyad, Marrakech, (Maroc), 1995.

9. E. Elqorachi and M. Akkouchi, On generalized d'Alembert and Wilson functional equations. Aequationes Math. 66(2003), No. 3, 241-256.

10. E. Elqorachi, M. Akkouchi, and A. Bakali, On Gel'fand elements of Banach algebras and related problems of harmonic analysis. Libertas Math. 15(1995), 107-118.

11. S. Helgason, Differential geometry and symmetric spaces. Pure and Applied Mathematics, XII. Academic Press, New York-London, 1962.

12. F. John, Plane waves and spherical means applied to partial differential equations. Interscience Publishers, New York-London, 1955.

13. M. A. Naimark, Normed algebras. Translated from the second Russian edition by Leo F. Boron. Third edition. Wolters-Noordhoff Series of Monographs and Textbooks on Pure and Applied Mathematics. Wolters-Noordhoff Publishing, Groningen, 1972.

14. H. SHIN'YA, Spherical matrix functions and Banach representability for locally compact motion groups. Japan. J. Math. (N.S.) 28(2002), No. 2, 163-201.

15. H. StetkæR, Functional equations and Spherical functions. Aarhus Univ. Preprint Series 18(1994).

16. H. StetkæR, D'Alembert's equation and spherical functions. Aequationes Math. 48 (1994), No. 2-3, 220-227.

17. H. StetkÆr, Wilson's functional equations on groups. Aequationes Math. 49(1995), No. $3,252-275$.

(Received 25.10.2003; revised 22.04.2004)

Authors' addresses:

Elhoucien Elqorachi

University of Ibnou Zohr

Faculty of Sciences

Department of Mathematics

Agadir, Morocco

E-mail: elqorachi@hotmail.com

Allal Bakali

University of Ibnou Tofail

Faculty of Sciences

Department of Mathematics

Kenitra, Morocco
Mohamed Akkouchi

University of Cadi Ayyad

Faculty of Sciences, Semlalia

Department of Mathematics

Marrakech, Morocco

E-mail: makkouchi@hotmail.com

Bouikhalene Belaid

University of Ibnou Tofail

Faculty of Sciences

Department of Mathematics

Kenitra, Morocco

E-mail: bbouikhalene@yahoo.fr 